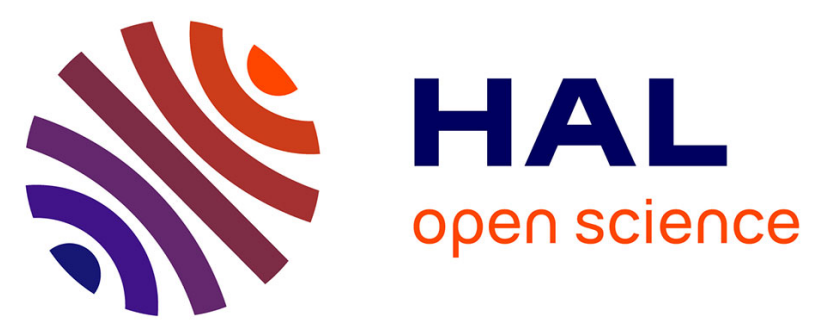

\title{
Couches minces supraconductrices à haute température critique: preparation in-situ de films orientés de YBa2CU3O7-X par depôt plasma
}

\author{
A. Perrin, Maryline Guilloux-Viry, Michael G. Karkut, J. Padiou, M. Sergent
}

\section{- To cite this version:}

A. Perrin, Maryline Guilloux-Viry, Michael G. Karkut, J. Padiou, M. Sergent. Couches minces supraconductrices à haute température critique : preparation in-situ de films orientés de YBa2CU3O7-X par depôt plasma. Journal de Physique III, 1991, 1 (2), pp.295-303. 10.1051/jp3:1991124 jpa-00248581

HAL Id: jpa-00248581

https://hal.science/jpa-00248581

Submitted on 1 Jan 1991

HAL is a multi-disciplinary open access archive for the deposit and dissemination of scientific research documents, whether they are published or not. The documents may come from teaching and research institutions in France or abroad, or from public or private research centers.
L'archive ouverte pluridisciplinaire HAL, est destinée au dépôt et à la diffusion de documents scientifiques de niveau recherche, publiés ou non, émanant des établissements d'enseignement et de recherche français ou étrangers, des laboratoires publics ou privés. 
Classification

Physics Abstracts

$74.10-74.30$

\title{
Couches minces supraconductrices à haute température critique : préparation in-situ de films orientés de $\mathrm{YBa}_{2} \mathrm{Cu}_{3} \mathrm{O}_{7-x}$ par dépôt plasma
}

\author{
A. Perrin ( $\left.{ }^{(}\right)$, M. Guilloux-Viry (1, 2), M. G. Karkut ( $\left.{ }^{1}\right)$, J. Padiou ( ${ }^{1}$ ) \\ et M. Sergent $\left({ }^{1}\right)$ \\ (1) Laboratoire de Chimie Minérale B, URA C.N.R.S. 254, Université de Rennes I, 35042 \\ Rennes Cedex, France \\ (2) CNET, Division OCM, Centre Lannion B, BP 40, 22301 Lannion Cedex, France
}

(Reģu le 16 mars 1990, révisé le 31 août 1990, accepté le 11 octobre 1990)

\begin{abstract}
Résumé. - Des couches minces du matériau supraconducteur $\mathrm{YBa}_{2} \mathrm{Cu}_{3} \mathrm{O}_{7-x}$ ont été préparées in situ par pulvérisation cathodique diode continue monocible sur des substrats monocristallins $(100) \mathrm{MgO},(100) \mathrm{SrTiO}_{3}$ et $(110) \mathrm{SrTiO}_{3}$ chauffés entre 600 et $700{ }^{\circ} \mathrm{C}$. L'orientation des films obtenus a été étudiée en fonction de la température du substrat. L'étude aux rayons $\mathrm{X}$ par application de la méthode du cristal tournant a permis d'observer le passage d'une croissance texturé à une croissance épitaxiale vers $700^{\circ} \mathrm{C}$ et de déterminer les relations d'épitaxie avec les divers substrats. Les films ont été caractérisés par mesures résistives et inductives. Les meilleurs échantillons, déposés sur $\mathrm{SrTiO}_{3}$, présentent des températures de début de transition résistive comprises entre 87 à $89 \mathrm{~K}$, des températures de fin de transition $(R=0)$ de 85 à $87 \mathrm{~K}$ et des densités de courant critique $(77 \mathrm{~K})$ de l'ordre de $10^{6} \mathrm{~A} \mathrm{~cm}^{-2}$. Les largeurs de transition, déterminées par la méthode inductive, sont inférieures à $1 \mathrm{~K}$. La réponse inductive est d'ailleurs fortement corrélée à la qualité des films et constitue une méthode de choix pour déterminer les conditions optimales de préparation.
\end{abstract}

\begin{abstract}
Superconducting thin-films of $\mathrm{YBa}_{2} \mathrm{Cu}_{3} \mathrm{O}_{7-x}$ material have been grown in situ by single target d.c. sputtering. Single-crystal (100) $\mathrm{MgO},(100) \mathrm{SrTiO}_{3}$ and (110) $\mathrm{SrTiO}_{3}$ substrates were heated between 600 and $700{ }^{\circ} \mathrm{C}$ and the film orientation has been studied as a function of substrate temperature. X-ray diffraction photographs, taken in a Weissenberg camera, were used to study the in-plane orientation of the films and to determine the epitaxial growth relations with the substrates. Films displayed only textured growth near $650{ }^{\circ} \mathrm{C}$ and epitaxial growth near $700{ }^{\circ} \mathrm{C}$. These thin-films have been measured by resistive and inductive techniques. Best samples, obtained on $\mathrm{SrTiO}_{3}$, exibit $T_{\mathrm{c}}$ onsets between 87 and $89 \mathrm{~K}, T_{\mathrm{c}}(R=0)$ between 85 and $87 \mathrm{~K}$ and critical current densities of about $10^{6} \mathrm{~A} \mathrm{~cm}^{-2}$ at $77 \mathrm{~K}$. The transition width of these samples, as determined from the inductive response, is less than $1 \mathrm{~K}$. We observed a clear correlation between the width of the a.c. inductive transition and the film quality and used this sensitive method for deposition-parameter optimization.
\end{abstract}

\section{Introduction.}

Un problème majeur concernant l'élaboration de couches minces d'oxydes supraconducteurs est lié à la nécessité, dans le cas de dépôts à froid, d'effectuer un post-traitement à haute 
température : à la température de cristallisation, de l'ordre de $900^{\circ} \mathrm{C}$, de fortes interactions chimiques film-substrat interviennent [1]. Une solution élégante apportée à cette limitation est la cristallisation in situ, sur substrat chauffé, pendant le dépôt [2]. Dans ce travail nous avons effectué une étude systématique de la croissance cristalline de couches minces de $\mathrm{YBa}_{2} \mathrm{Cu}_{3} \mathrm{O}_{7-x}$, déposées sur divers substrats, en fonction de la température de dépôt dans le but d'accéder à des films épitaxiès, condition indispensable pour obtenir de fortes densités de courant critique, nécessaires à de nombreuses applications potentielles, y compris en microélectronique. La détermination précise de la texture de tels films présente une importance considérable afin de faire la distinction entre un film simplement texturé (un axe cristallographique orienté par rapport au substrat) et un film véritablement épitaxié (les trois axes cristallographiques orientés par rapport au substrat). Les méthodes habituellement utilisées ne sont pas toujours facilement accessibles (diffraction d'électrons de haute énergie RHEED, figures de pôles, spectroscopie Rutherford) et parfois destructives (diffraction électronique en transmission après amincissement du substrat). Dans ce travail, nous montrons l'apport d'une méthode aussi classique et peu onéreuse que l'enregistrement des clichés de diffraction $\mathrm{X}$ de cristal tournant pour accéder à cette donnée et nous avons établi une corrélation entre la texture de nos films et leurs propriétés magnétiques et de transport.

\section{Partie expérimentale.}

Les films ont été déposés sur des substrats monocristallins (100) $\mathrm{MgO},(100) \mathrm{SrTiO}_{3}$ et (110) $\mathrm{SrTiO}_{3}$ par pulvérisation cathodique monocible diode continue dans des conditions dérivées de celles que nous avons définies précédemment pour la croissance ex situ de films du matériau $\mathrm{YBa}_{2} \mathrm{Cu}_{3} \mathrm{O}_{7-x}[1]$ : nous avions en particulier montré que l'utilisation d'une pression de dépôt élevée et d'une faible tension de polarisation permettait de réduire les problèmes de stœchiométrie des films liés à une rétropulvérisation sélective. L'efficacité de cette procédure a été confirmée par une étude systématique indépendante [3]. Cependant la fonction de transfert est sensiblement modifiée par rapport aux dépôts à froid, en relation avec la nécessité d'utiliser un plasma enrichi en oxygène afin d'oxyder le film en cours de croissance. En outre, la vitesse de dépôt chute considérablement, de $250 \mathrm{~nm} \cdot \mathrm{h}^{-1}$ à $50 \mathrm{~nm} \cdot \mathrm{h}^{-1}$ environ. Les paramètres de dépôt utilisés dans ce travail sont regroupés dans le tableau I.

Tableau I. - Conditions typiques de dépôt.

\begin{tabular}{|l|l|}
\hline Polarisation de la cible & $-180 \mathrm{à}-250 \mathrm{~V}$ \\
Distance cible-substrat & $15-19 \mathrm{~mm}$ \\
Pression totale & $0,4-1,5 \mathrm{mbar}$ \\
Teneur en oxygène & $3-15 \%$ \\
Durée du presputtering & $1-2 \mathrm{~h}$ \\
Epaisseur des films & $500-2000 \AA$ \\
\hline
\end{tabular}

Les films ont été caractérisés structuralement par diffraction de rayons $\mathrm{X}$ en mode standard $\Theta-2 \Theta$ (rayonnement $\mathrm{CuK} \bar{\alpha}$ ) et par la méthode du cristal tournant (chambre de Weissenberg $\varnothing 180 / 2 \pi \mathrm{mm}$ ). Leur état de surface a été déterminé par microscopie électronique à balayage et leur composition par microanalyse EDS. Les mesures résistives ont été effectuées par la méthode des quatre points (contacts à la laque d'argent) dans une canne de mesure refroidie à l'azote liquide (éventuellement pompé). Les mesures inductives ont été réalisées à l'aide d'un dispositif spécifique construit au laboratoire : la cellule comporte un enroulement primaire et 
deux micro-bobines secondaires enroulées sur des barreaux de ferrite, la couche étant insérée entre le champ primaire et les bobines dé détection. L'expulsion de flux au moment de la transition déséquilibre un pont d'inductance mutuelle classique travaillant à $119 \mathrm{~Hz}$.

\section{Résultats.}

2.1 CRoissance CRISTAlline DEs Films. - Les diagrammes de rayons $\mathrm{X}$ de films déposés sur (100) MgO montrent que leur texture évolue considérablement [4] dans un domaine très étroit de la température $T_{\mathrm{s}}$ du dépôt : la cristallisation débute aux environs de $600{ }^{\circ} \mathrm{C}$. La figure 1 montre qu'à plus haute température les couches minces prennent d'abord une orientation aléatoire, puis une orientation $h 00\left(T_{\mathrm{s}}=630^{\circ} \mathrm{C}\right)$. Un début d'orientation 001 apparaît à $640^{\circ} \mathrm{C}$ et, dès $650^{\circ} \mathrm{C}$, les couches minces sont préférentiellement orientées 001 . Un balayage en $\Theta$ avec $2 \Theta$ fixé aux environs de $38^{\circ}$ sur le centre du pic 005 ( « rocking curve ») montre que l'écart entre la direction $c$ des cristallites du film et la normale au plan du substrat ne dépasse pas une fraction de degré.

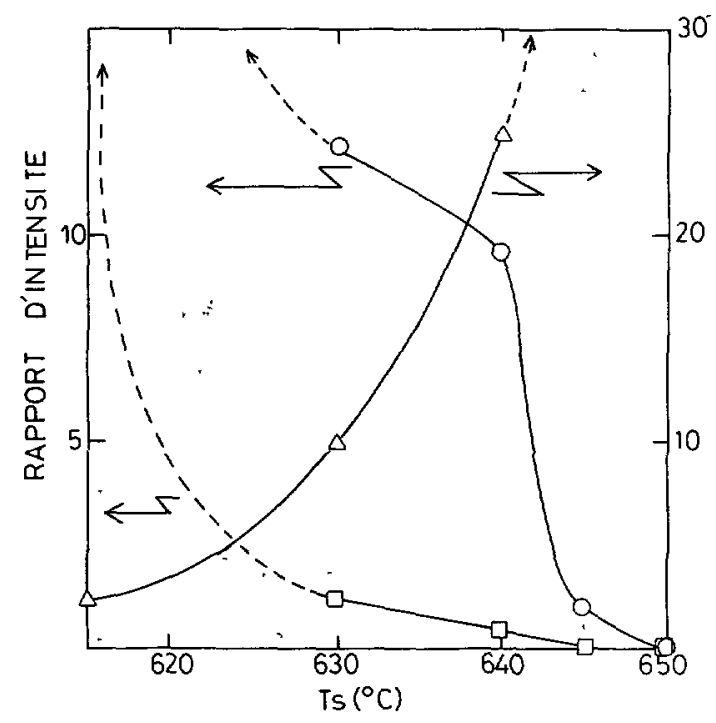

Fig. 1. - Rapport des intensités de pics de diffraction $\mathrm{X}$ caractéristiques en fonction de la température de substrat pour des films déposés sur $(100) \mathrm{MgO}:(\mathrm{O}) I_{200} / I_{005}$; (口) $I_{(103+110)} / I_{005} ;(\Delta) I_{200} / I_{(103+110)}$. [Intensity ratio of characteristic XRD reflections as a function of substrate temperature for thin-films deposited on (100)MgO : (O) $I_{200} / I_{005}$; (ㅁ) $\left.I_{(103+110)} / I_{005} ;(\Delta) I_{200} / I_{(103+110)}\right]$

Cependant le diagramme de cristal tournant [5] de cette couche (Fig. 2a) met en évidence des anneaux de diffraction ce qui prouve que les axes $a$ et $b$ du film sont orientés de manière aléatoire dans le plan du substrat. Par contre, pour des températures de dépôt légèrement supérieures, $680-700^{\circ} \mathrm{C}$, les couches minces deviennent quasi-monocristallines, avec les axes $a$ et $b$ parallèles aux axes du substrat : la figure $2 b$ met en évidence des rangées de taches de diffraction parfaitement alignées avec celles de MgO. L'absence de tout élargissement significatif des taches de diffraction de la couche par rapport à celles du substrat montre que l'effet mosaïque dans ces directions est inférieur au degré. L'espacement des rangées permet de déterminer la valeur des paramètres $a$ et/ou $b: 0,385 \mathrm{~nm}$. 


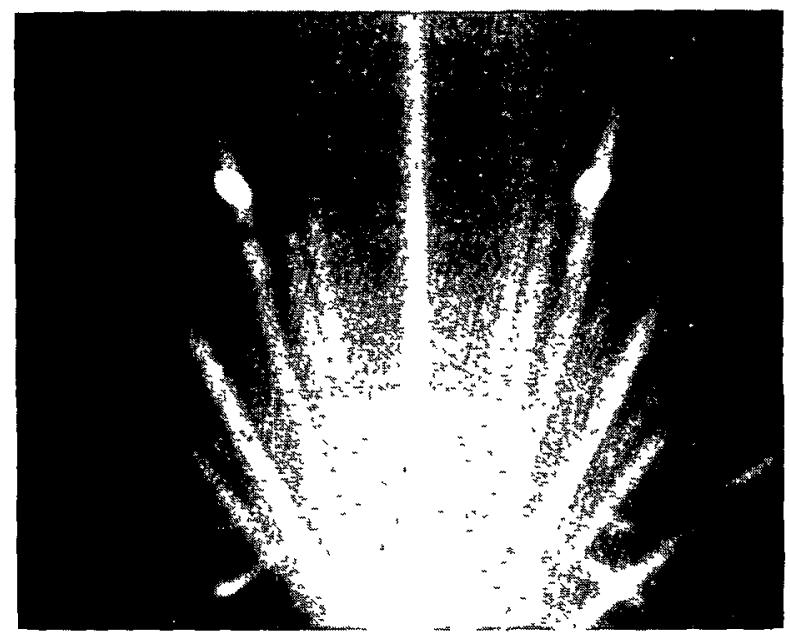

a)

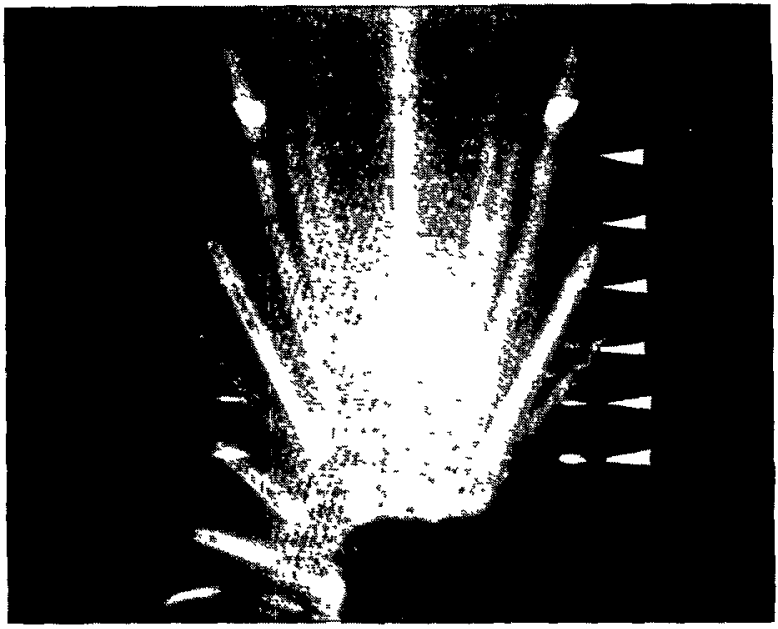

b)

Fig. 2. - Clichés de diffraction $\mathrm{X}$ (cristal oscillant) de deux couches minces YBaCuO déposées in situ sur (100)MgO. a) film déposé à $650^{\circ} \mathrm{C}$, orienté préférentiellement $00 \ell$. Noter la présence d'anneaux, caractéristiques d'une désorientation dans le plan $(a, b) ; b)$ film déposé à $690^{\circ} \mathrm{C}$, orienté préférentiellement $00 \ell$. Noter les points de diffraction du film (strate 0 et strate $1:$ flèches blanches), caractéristiques d'une orientation des axes cristallographiques $a$ et $b$ du film colinéaires à ceux du substrat.

[X-ray diffraction photographs (oscillating crystal) of two thin-films deposited in situ on (100)MgO; a) film deposited at $650{ }^{\circ} \mathrm{C}$, with $00 \ell$ preferred orientation. Note the diffraction rings, characteristic of disorder in the $(a, b)$ plane; b) film deposited at $690{ }^{\circ} \mathrm{C}$. Note the discrete diffraction dots of the film (diffraction layers 0 and 1 : white arrows), characteristic of a perfect alignment of the $a$ and $b$ axes of the film with cristallographic axes of the substrate.]

La nature du substrat a également une influence considérable sur la texture de ces films. Ainsi pour une même séquence de dépôt à $645^{\circ} \mathrm{C}$ sur (100) $\mathrm{MgO},(100) \mathrm{SrTiO}_{3}$ et (1102) $\mathrm{Al}_{2} \mathrm{O}_{3}$ monocristallins, on obtient une orientation aléatoire sur le saphir et une 
orientation principalement $00 \ell$ sur $\mathrm{MgO}$. Sur $\mathrm{SrTiO}_{3}$ le film, orienté $00 \ell$ et éventuellement h00 présente encore une faible cristallinité : la croissance de films texturés sur ce type de substrats nécessite une température de dépôt d'environ $10-20{ }^{\circ} \mathrm{C}$ supérieure à celle mise en auvre pour $\mathrm{MgO}$. Des films entièrement orientés $00 \ell$ (sans trace d'orientation h00) sont obtenus à une température de dépôt supérieure à $700^{\circ} \mathrm{C}$. Des dépôts ont été également effectués sur (110) $\mathrm{SrTiO}_{3}$ dans le même domaine de température: les diagrammes de diffraction $\Theta-2 \Theta$ montrent que ces.films sont totalement orientés, soit (103) soit (110). Les clichés de cristal tournant ont permis de lever cette ambiguité due à un angle de diffraction identique pour les réflexions 103 et 110 : ils ont prouvé l'orientation (103) avec les relations d'épitaxie suivantes : $(103)_{\text {Film }} / /(110)_{\text {Substrat }}$ et $|010|_{\text {Film }} / /|001|_{\text {Substrat }}[5]$. Ce type d'épitaxie a été observé indépendamment par enregistrement de figures de pôle [6] et par RHEED [7], tandis que l'orientation (110) apparaît spécifiquement à plus basse température [7].

2.2 RÉSistivitÉ ÉleCTRIQUE. - Les mesures résistives des films déposés à diverses températures sur (100)MgO mettent en évidence une forte corrélation entre leur comportement et leur texture (Fig. 3). En particulier, le coefficient de température (pente de la courbe de résistivité dans l'état normal) augmente constamment avec la température de dépôt, avec une variation importante dans le domaine $640-645^{\circ} \mathrm{C}$, correspondant à l'apparition de l'orientation privilégiée 001 . Le début de transition, observé à partir de $630^{\circ} \mathrm{C}$, apparaît à une température constante $T_{\mathrm{c}_{\text {onset }}}=88 \mathrm{~K}$. Pour cette série d'échantillons, la fin de transition n'est pas observée au-dessus de $77 \mathrm{~K}$ en raison d'une oxygénation insuffisante. Dans une seconde étape, les conditions de dépôt et d'oxygénation ont été optimisées, en particulier par augmentation de la pression d'oxygène dans le plasma et par le contrôle de celle-ci au cours

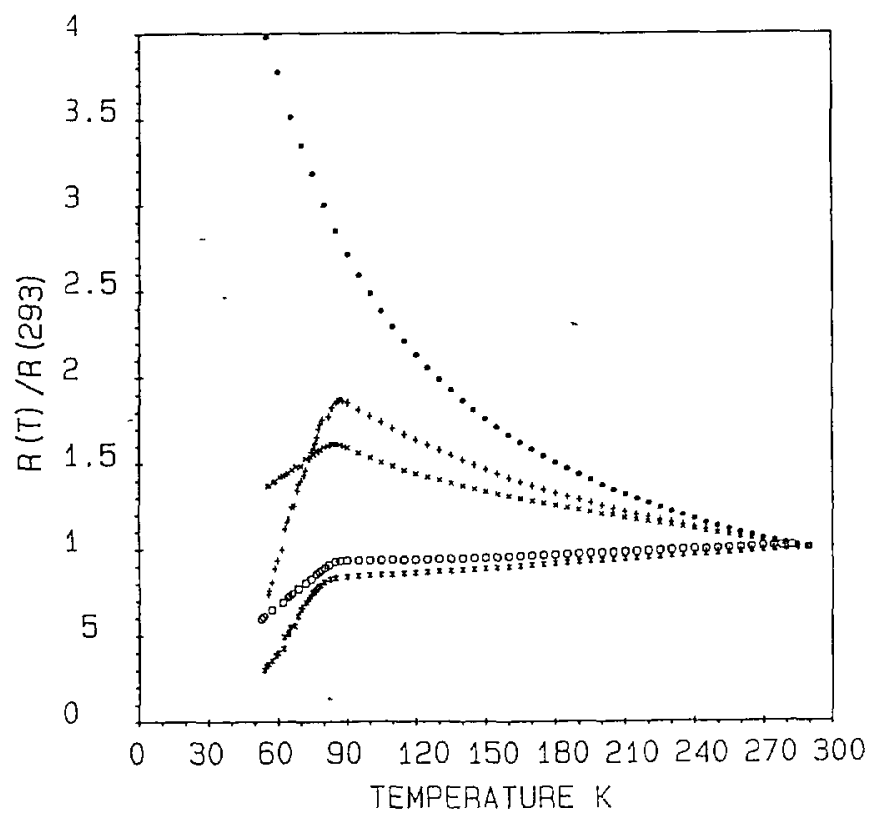

Fig. 3. - Comportement résistif pour divers films, en fonction de la température du substrat: (O) $615^{\circ} \mathrm{C} ;(+) 630^{\circ} \mathrm{C} ;(\times) 640{ }^{\circ} \mathrm{C}$; (口) $645{ }^{\circ} \mathrm{C} ;\left(\right.$ (*) $^{\circ} 650^{\circ} \mathrm{C}$.

[Resistive behaviour of thin films deposited at various temperatures : (O) $615^{\circ} \mathrm{C} ;(+) 630^{\circ} \mathrm{C} ;(x)$ $640{ }^{\circ} \mathrm{C}$; (D) $645{ }^{\circ} \mathrm{C} ;\left(^{*}\right) 650{ }^{\circ} \mathrm{C}$. $]$ 
du refroidissement [8]. Cette procédure a permis d'obtenir de façon reproductible des films 001 de bonne 'qualité, avec des résistances nulles au-delà de $80 \mathrm{~K}$. Les meilleurs résultats obtenus à ce jour sont résumés sur la figure 4 : ce film, déposé sur (100) $\mathrm{SrTiO}_{3}$, présente un rapport de résistivité dans l'état normal $R(290 \mathrm{~K}) / R(100 \mathrm{~K})$ de 2,85 , une résistance nulle à $86,4 \mathrm{~K}$, avec une largeur de transition (10-90\%) de $1,3 \mathrm{~K}$. Une première évaluation de la densité de courant critique, obtenue à partir de la densité de courant provoquant l'apparition d'une tension (critère $5 \mu \mathrm{V} \mathrm{cm}{ }^{-1}$ ) ' pour un échantillon non gravé, a conduit à une valeur de l'ordre de $10^{6} \mathrm{~A} \mathrm{~cm}^{-2}$ à $77 \mathrm{~K}$. Il n'a pas été effectué de corrections tenant compte de la distribution du courant; cependant, des mesures plus récentes effectuées avant et après gravure sur d'autres films ont confirmé la validité de ces premiers résultats.

Le début de transition, à $89-90 \mathrm{~K}$, est, de façon systématique, légèrement plus élevé dans le cas de substrats de $\mathrm{SrTiO}_{3}$ que dans celui de $\mathrm{MgO}$ : cette différence pourrait être liée à une légère diffusion de $\mathrm{MgO}$ dans le film [1].

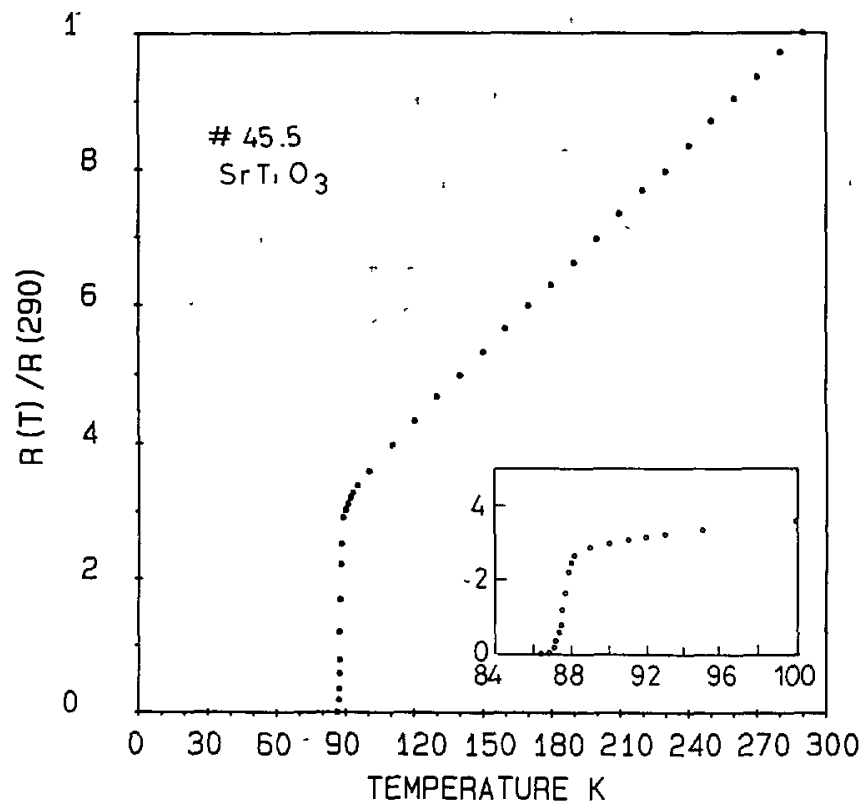

Fig. 4. - Variation thermique de la résistivité relative d'un film épitaxié sur (100) $\mathrm{SrTiO}_{3}$. Noter le comportement linéaire dans l'état normal et son extrapolation à zéro.

[Thermal variation of the relative resistivity for a film epitaxially grown on (100)SrTiO ${ }_{3}$. Note the linear behaviour in the normal state and the intercept close to zero.]

2.3 SusCePTIBILITÉ MAGNÉTIQUE. - Les mesures de susceptibilité alternative [8] ont fourni des informations complémentaires importantes sur la transition supraconductrice et la qualité de l'échantillon, notamment en ce qui concerne son homogénéité. La figure 5 représente la variation en température, au voisinage de la transition, des parties réelle $\chi^{\prime}$ et imaginaire $\chi$ " de la susceptibilité pour l'échantillon de la figure 4. L'étroitesse de $\chi_{(T)}^{\prime}$ et l'absence de tout épaulement ainsi que le pic unique et aigu en $\chi^{\prime \prime}$ (largeur à mi-hauteur d'environ $0,6 \mathrm{~K}$ ) sont l'indice d'une très bonne cristallisation (absence d'effet -de granularité) de ce film et d'une excellente homogénéité sur toute sa surface. 


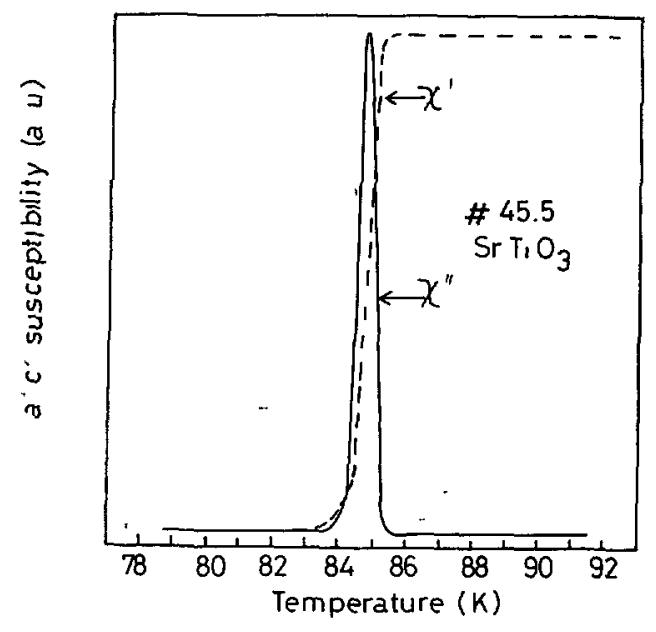

Fig. 5. - Réponse inductive, au voisinage de la tränsition supraconductrice, du film de la figure 4.

[Inductive reponse, near the superconducting transition, of the same film as in figure 4.]

2.4 MoRPhologie DE SURFACE: - L'état de surface des films est le troisième critère important, avec la température critique et la densité de courant critique, pour une future utilisation en microélectronique, en relation avec les opérations de lithogravure. L'observation au microscope électronique à balayage [4] de films déposés sur $\mathrm{MgO}$ montre qu'ils sont extrêmement lisses : une vue oblique d'une fracture obtenue par clivage du substrat permet d'estimer la rugosité à moins de $30 \mathrm{~nm}$. La plupart des films présentent encore quelques cristaux sub-microniques de $\mathrm{CuO}$ disséminés à leur surface, en relation avec une légère surstœechiométrie en cuivre, confirmée par la microanalyse. Ces cristaux sont expulsés de la couche au cours de la croissance et ne détériorent pas les propriétés supraconductrices. La spectroscopie RBS confirme que le film conserve la stœchiométrie $1: 2: 3$ et que l'excès de cuivre reste localisé en surface [9]. De ce point de vue, cette situation est beaucoup plus favorable qu'une sous-stcehiométrie, même légère, qui entraîne la formation de phases isolantes telles que $\mathrm{Y}_{2} \mathrm{BaCuO}_{5}$ et $\mathrm{BaCuO}_{2}$ qui semblent au contraire s'accumuler aux joints de grains: une telle accumulation a d'ailleurs été observée directement par microscopie électronique en transmission [10] et indirectement par spectroscopie Raman [11]. Bien entendu, la présence de ces dernières phases conduit alors à la formation de liaisons faibles ("weak links ») rendant impossible l'obtention de densités de courant critique élevées. Cette dernière observation est également en accord avec les données de Y. Shirakawa [12] qui ont mis en évidence l'importance de la valeur du rapport $\mathrm{Y} / \mathrm{Ba}$.

\section{Discussion et conclusion.}

L'utilisation d'un système simple de pulvérisation cathodique diode continue monocible nous a permis d'obtenir in situ, de façon réproductible, des films de haute qualité du matériau $\mathrm{YBa}_{2} \mathrm{Cu}_{3} \mathrm{O}_{7-x}^{\prime}$. les films déposés sur (100) $\mathrm{SrTiO}_{3}$ ont habituellement dés débuts de transition résistive compris entre 87 et $89 \mathrm{~K}$ et des fins de transition entre 84 et $86 \mathrm{~K}$, avec des largeurs de transition inférieures à $1 \mathrm{~K}$ par mesure inductive. Des résultats presque aussi bons ont été obtenus sur les substrats de $(100) \mathrm{MgO}$, puisque la seule différence essentielle est un abaissement de la température critique d'environ 2 à $3 \mathrm{~K}$ : on devait pourtant s'attendre à une certaine dégradation des propriétés supraconductrices par diffusion du magnésium [1] mais 
cette dernière reste très limitée et n'a pu être mise en évidence de façon certaine par RBS en raison de la présence de pics supplémentaires dus aux isotopes 25 et 26 de $\mathrm{Mg}$ [9].

La méthode du cristal tournant, utilisant une chambre de Weissenberg d'emploi simple et courant, s'est révélée un outil très performant pour étudier la structure cristalline des films dans le plan du substrat, permettant d'une part d'optimiser les températures de dépôt, d'autre part de déterminer sans ambiguïté les relations d'épitaxie. Nous avons ainsi montré, par exemple, un parfait alignement des axes cristallographiques $a$ et/ou $b$ du film (indiscernables en raison du mâclage habituel dans ce matériau) avec les axes du substrat quand la température de dépôt avoisine $700^{\circ} \mathrm{C}$. Il n'est évidemment pas possible de faire la distinction entre un film strictement monocristallin (abstraction faite des mâcles) et un film constitué d'un assemblage de petits monocristaux parfaitement alignés. L'observation au microscope électronique à balayage le long de lignes de fracture du film, obtenues par clivage du substrat de $\mathrm{MgO}$, est plutôt favorable à la seconde hypothèse, puisqu'elle montre la présence de blocs d'une centaine de nanomètres d'arête; cependant, ces blocs deviennent très difficiles à discerner dès que l'on s'écarte de quelques microns de la ligne de fracture et il n'est pas impossible que leur formation soit liée à des relâchements de contraintes mécaniques au moment du clivage du substrat.

La caractérisation magnétique, mettant à profit l'expulsion de flux dans un montage d'inductance mutuelle, est extrêmement utile car elle est rapide, non destructive et donne des informations essentielles sur la qualité des films : elle est en particulier beaucoup plus sensible à l'homogénéité des films que la mesure résistive $R(T)$. Ainsi nous avons montré [8] que deux films qui présentaient des transitions résistives identiques avaient des comportements inductifs très différents, avec des largeurs à mi-hauteur de $\chi^{\prime \prime}$ dans un rapport 10. Nous avons mis en évidence une corrélation très nette entre la largeur du pic en $\chi^{\prime \prime}$ et la densité de courant critique, apparemment reliée à l'influence des joints de grains sur la cohérence de l'état supraconducteur. Des observations similaires ont été rapportées très récemment par Neumann et al. [13]. L'étude comparative pour diverses couches minces, de leur cycle d'hystérésis magnétique (déterminé à l'aide d'un magnétomètre à SQUID) et de leur réponse en susceptibilité alternative est actuellement en cours. Les résultats obtenus confirment clairement cette corrélation [14] : la méthode inductive constitue donc un outil particulièrement performant pour l'optimisation des conditions d'élaboration de ces films supraconducteurs.

Il faut enfin souligner que nos films $00 \ell$ réalisés in-situ présentent une bonne conservation dans le temps sans nécessiter de passivation. Ainsi un film déposé sur (100) $\mathrm{MgO}$ a été sélectionné pour sa qualité relativement médiocre $\left(T_{\mathrm{c}}(R=0)=77 \mathrm{~K}\right)$ le rendant a priori plus sensible au vieillissement : après avoir été exposé à l'air ambiant pendant 2 mois puis gravé chimiquement, il présentait toujours le même comportement résistif. De même, une densité de courant critique de $3 \times 10^{5} \mathrm{~A} \mathrm{~cm}^{-2}$ à $77 \mathrm{~K}$ a été déterminée par méthode résistive après gravure ionique sur un échantillon $(R=0$ à $81 \mathrm{~K})$ conservé à l'air durant 2 mois : la valeur de $J_{\mathrm{c}}$ obtenue reste tout à fait comparable à la valeur initiale estimée pour cet échantillon.

\section{Remerciements.}

Ce travail a été soutenu financièrement par le CNET Lannion B (Contrat 89 8B 054) et le

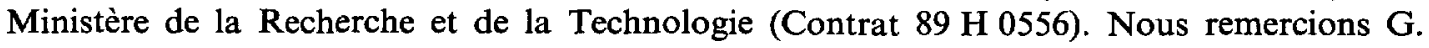
Dousselin, Y. Pellan, J. Pinel et Mme F. Thoumyre (Département de Physique de l'INSA de Rennes, URA CNRS 786) pour les opérations de gravure ionique et chimique et les mesures d'épaisseur des films et $O$. Peña pour les mesures inductives. 


\section{Bibliographie}

[1] Perrin A., Li Z. Z., Peña O., Padiou J. et Sergent M., Revue Phys. Appl. 23 (1988) 257.

[2] Bando Y., Terashima T., Iujima K., Yamamoto K. et Mazaki H., Physica C 153-155 (1988) 810.

[3] Li H. C., Linker G., Ratzel F., Smithey R. et Geerk J., Appl. Phys. Lett. 52 (1988) 1098.

[4] Guilloux-Viry M., Karkut M. G., Perrin A. et Sergent M., Mater. Lett. 10 (1990) 126.

[5] Perrin A., Guilloux-Viry M., Karkut M. G. et Sergent M., Appl. Phys. Lett. (1991) sous presse.

[6] Triscone J. M., Communication privée.

[7] Terashima T., Bando Y., Iijima K., Yamamoto K. et Hirata K., Appl. Phys. Lett. 53 (1988) 2232.

[8] Guilloux-Viry M., Karkut M. G., Perrin A., Peña O., Padiou J. et Sergent M., Physica C 166 (1990) 105.

[9] Kechouane M., L'Haridon H., Favennec P. N., Guilloux-Viry M., Karkut M. G. et PERRIN A., Travaux non publiés.

[10] Brokman A., Palhan M. et Weger M., Physica C 153-155 (1988) 1653.

[11] Erle A., Blumenröder S. et GUntherodt G., Physica C 162-164 (1989) 1093.

[12] Shirakawa Y. et Kobayashi M., Jpn. J. Appl. Phys. 28 (1989) L1405.

[13] Neumann C., Ziemann P., Geerk J. et Li X. X., Physica C 162-164 (1989) 321.

[14] GuillouX-Viry M., Thèse d'Université, Rennes (23 janvier 1991). 